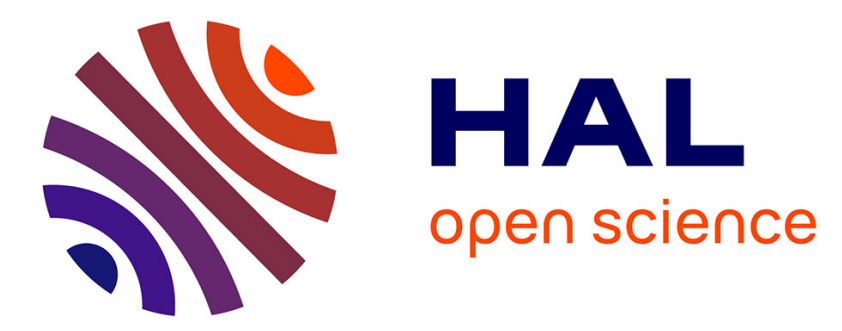

\title{
What can be learned from a proto-neutron star's mass and radius?
}

\author{
Edwan Preau, Aurélien Pascal, Jérôme Novak, Micaela Oertel
}

\section{To cite this version:}

Edwan Preau, Aurélien Pascal, Jérôme Novak, Micaela Oertel. What can be learned from a protoneutron star's mass and radius?. Monthly Notices of the Royal Astronomical Society, 2021, 505 (1), pp.939-946. 10.1093/mnras/stab1348 . hal-03123354

\section{HAL Id: hal-03123354 \\ https://hal.science/hal-03123354}

Submitted on 27 Jan 2021

HAL is a multi-disciplinary open access archive for the deposit and dissemination of scientific research documents, whether they are published or not. The documents may come from teaching and research institutions in France or abroad, or from public or private research centers.
L'archive ouverte pluridisciplinaire HAL, est destinée au dépôt et à la diffusion de documents scientifiques de niveau recherche, publiés ou non, émanant des établissements d'enseignement et de recherche français ou étrangers, des laboratoires publics ou privés. 


\title{
What can be learned from a proto-neutron star's mass and radius?
}

\author{
E. Préau, ${ }^{1,2 \star}$ A. Pascal, ${ }^{1} \dagger$ J. Novak ${ }^{1} \ddagger$ and M. Oertel ${ }^{1} \S$ \\ ${ }^{1}$ Laboratoire Univers et Théories, Observatoire de Paris, Université PSL, CNRS, Université de Paris, 92190 Meudon, France \\ ${ }^{2}$ Astroparticule et Cosmologie, Université de Paris, CNRS, 75013 Paris, France
}

27 January 2021

\begin{abstract}
We make extensive numerical studies of masses and radii of proto-neutron stars during the first second after their birth in core-collapse supernova events. We use a quasi-static approach for the computation of proto-neutron star structure, built on parameterized entropy and electron fraction profiles, that are then evolved with neutrino cooling processes. We vary the equation of state of nuclear matter, the proto-neutron star mass and the parameters of the initial profiles, to take into account our ignorance of the supernova progenitor properties. We show that if masses and radii of a proto-neutron star can be determined in the first second after the birth, e.g. from gravitational wave emission, no information could be obtained on the corresponding cold neutron star and therefore on the cold nuclear equation of state. Similarly, it seems unlikely that any property of the proto-neutron star equation of state (hot and not beta-equilibrated) could be determined either, mostly due to the lack of information on the entropy, or equivalently temperature, distribution in such objects.
\end{abstract}

Key words: stars: neutron - equation of state - methods: numerical

\section{INTRODUCTION}

Neutron stars are fascinating objects as their understanding combines many aspects of physics under extreme conditions: relativistic gravitational field and matter at supra-nuclear densities, to list the most notable ones (for a more detailed description, see e.g. Haensel et al. 2007). They can therefore be used as astrophysical laboratories to test our understanding of the laws of physics under conditions which cannot be reached by Earth-based experiments. Whereas the theory of General Relativity is tested to a high accuracy within the neutron star context (Wex \& Kramer 2020; Kramer et al. 2006), the situation is not so favorable when probing the properties of nuclear matter. In this respect, one of the major objectives in the field is the determination of the equation of state (EoS) of cold nuclear matter in beta-equilibrium, relating e.g. pressure $P$ and baryon number density $n_{b}$. This EoS is relevant for the description of rather old (more than a few minutes) neutron stars at the end of their chemical evolution, i.e. in equilibrium with respect to weak interactions and transparent to neutrinos. Recent progress has been made with the first detection of gravitational waves (GW) from a binary neutron star coalescence, which has put some constraints on the neutron star tidal deformability (Abbott et al. 2018). This

\footnotetext{
* E-mail: edwan-preau@hotmail.fr

† E-mail: aurelien.pascal@obspm.fr

$\ddagger$ E-mail: jerome.novak@obspm.fr

$\S$ E-mail: micaela.oertel@obspm.fr
}

first detection shows the potential of future GW detections from binary neutron star mergers to constrain the EoS, see e.g. (De et al. 2018; Dexheimer et al. 2019; Capano et al. 2020; Malik et al. 2019; Güven et al. 2020). There exists in fact a one-to-one relation between a given EoS and the tidal deformability as function of the star's mass (Hinderer et al. 2010) similar to the mass-radius diagram (M-R diagram) of cold non-rotating neutron stars. The latter is the subject of the standard approach for inferring constraints on the nuclear matter EoS (Ozel et al. 2010; Steiner et al. 2010, 2013a; Ozel et al. 2016). Therefore, many efforts are underway to measure at least some points in this diagram, see for example recent results by NICER (Miller et al. 2019; Riley et al. 2019).

In a prospective way, Torres-Forné et al. (2019a), have numerically determined empirical relations between masses $\left(M_{\mathrm{PNS}}\right)$, radii $\left(R_{\mathrm{PNS}}\right)$ and oscillation frequencies of protoneutron stars (PNS). The PNS forms during core-collapse supernova (CCSN) events, which should be good sources of gravitational radiation, too. The detection of GW from such modes could thus directly be related to PNS properties. With a numerical perturbative approach in General Relativity Torres-Forné et al. (2018, 2019b) have identified oscillation modes of a newly born PNS during the first second of its life and related them to the PNS surface gravity $\left(M_{\mathrm{PNS}} / R_{\mathrm{PNS}}^{2}\right)$ for so-called $g$-modes and shock properties for $p$-modes. With some additional assumptions, it is then possible to deduce $M_{\mathrm{PNS}}$ and $R_{\mathrm{PNS}}$ from observed mode frequencies in GW. However, the structure of newly born PNSs during the first seconds of their life is very different from that 
$[\mathrm{h}]$
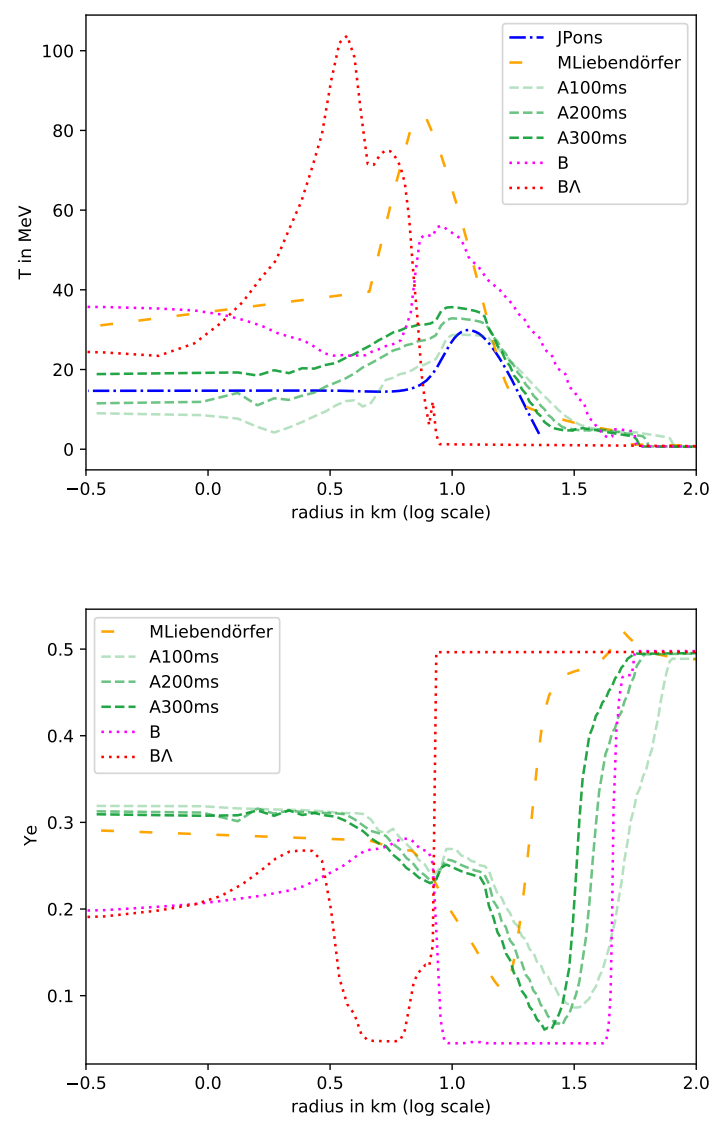

Figure 1. Temperature $T$ (top) and electron fraction $Y_{e}$ (bottom) radial profiles obtained from various core-collapse supernova simulations (see text for details).

of cold neutron stars (Prakash et al. 1997; Pons et al. 1999). In particular, properties of nuclear matter cannot in general be cast into a simple function of one variable as $P\left(n_{b}\right)$, but usually rely on a more complex 3 -dimensional relation such as $P\left(n_{b}, T, Y_{e}\right)$, with $T$, the temperature and $Y_{e}=\left(n_{e}-n_{\bar{e}}\right) / n_{b}$ the electron fraction, i.e. the ratio between electron number density $\left(n_{e}\right.$, minus the positron number density $\left.n_{\bar{e}}\right)$ and $n_{b}$.

In this context, the aim of this work is to investigate two problems. (P1) how much would the determination of the mass and radius of a PNS born in core-collapse supernovae be able to constrain the M-R diagram of cold neutron stars, and therefore also their cold equation of state $P\left(n_{b}\right)$ ?

The second one (P2) is related to the first one: is it possible, with such observations, to constrain at least the (3dimensional) EoS of hot newly born PNS?

We present the model and the methods that were used to tackle these two problems in Sec. 2. Our results are presented in Sec. 3, before some concluding remarks in Sec. 4.

\section{QUASI-STATIC APPROACH TO PROTO-NEUTRON STAR EVOLUTION}

In this work, we shall only consider models in spherical symmetry, i.e. non-rotating, non-magnetized PNS, and we develop in this section a quasi-static approach to derive their structure. Although a PNS is an evolving star through cooling processes, the quasi-static hypothesis is justified by the fact that the timescale necessary to establish hydrodynamic equilibrium is much shorter than the PNS evolution timescale, mostly determined by cooling through neutrino emission (Pons et al. 1999). Following this work, we consider models in hydrostatic equilibrium, meaning that we follow the evolution of the PNS driven by the neutrino cooling processes, but at each time-step, we solve for hydrostatic equilibrium. It is in particular assumed here that the eventual supernova explosion is successful, so that matter outside the shock is excised from the numerical grid, once this shock has crossed the matter of the PNS (see the discussion e.g. by Roberts \& Reddy 2017).

The starting point is the expression for the metric of a static, spherically symmetric spacetime, using the standard Schwarzschild-type coordinates:

$\mathrm{d} s^{2}=-c^{2} \mathrm{~d} t^{2} \mathrm{e}^{2 \Phi}+\mathrm{e}^{2 \lambda} \mathrm{d} r^{2}+r^{2}\left(\mathrm{~d} \theta^{2}+\sin ^{2} \theta \mathrm{d} \phi^{2}\right)$,

where $\Phi(r)$ and $\lambda(r)$ are the two gravitational potentials. It is often more convenient to introduce a new function $m(r)$ such that:

$\mathrm{e}^{-\lambda}=\sqrt{1-2 G m /\left(r c^{2}\right)}$.

In the Newtonian limit, $m(r)$ represents the gravitational mass inside a sphere with radial coordinate $r$. The total gravitational mass of the star $M_{g}=m(R)$ is the quantity that appears in the Schwarzschild solution for the metric outside the star. Note that it is this mass that could be determined through GW observation of $p$ - and $g$-modes $\left(M_{\mathrm{PNS}}=M_{g}\right)$. Determining the structure of a star amounts to deriving the stress-energy tensor field $T_{\mu \nu}$, related to the metric in Eq. (1) through Einstein Equations:

$\mathcal{R}_{\mu \nu}-\frac{1}{2} g_{\mu \nu} \mathcal{R}=\frac{8 \pi G}{c^{4}} T_{\mu \nu}$,

where $\mathcal{R}_{\mu \nu}$ is the Ricci tensor of the metric and $\mathcal{R}=g^{\mu \nu} \mathcal{R}_{\mu \nu}$ the Ricci scalar.

We then assume the neutron star matter to be made of a single perfect fluid and neglect any other contribution to the stress-energy tensor: $T_{\mu \nu}=(P+\mathcal{E}) u_{\mu} u_{\nu}+P g_{\mu \nu}$, where $u_{\mu}$ is the 4 -velocity of matter, $\mathcal{E}$ the total energy density and $P$ the pressure. This approximation is justified for newly born neutron stars because shear stresses produced by strong magnetic fields are generally negligible compared with the pressure. On the other hand, the PNS is hot: crust is not formed yet and superfluid effects are not present, either.

Einstein equations (3) in this case yield the well known Tolman-Oppenheimer-Volkov (TOV) system for hydrostatic equilibrium for a static spherically symmetric star:

$$
\begin{aligned}
\frac{\mathrm{d} m}{\mathrm{~d} r} & =\frac{4 \pi}{c^{2}} r^{2} \mathcal{E}, \\
\frac{\mathrm{d} \Phi}{\mathrm{d} r} & =\frac{G m}{r^{2} c^{2}}\left(1+\frac{4 \pi P r^{3}}{m c^{2}}\right)\left(1-\frac{2 G m}{c^{2} r}\right)^{-1}, \\
\frac{\mathrm{d} P}{\mathrm{~d} r} & =-(\mathcal{E}+P) \frac{\mathrm{d} \Phi}{\mathrm{d} r},
\end{aligned}
$$


$[\mathrm{h}]$
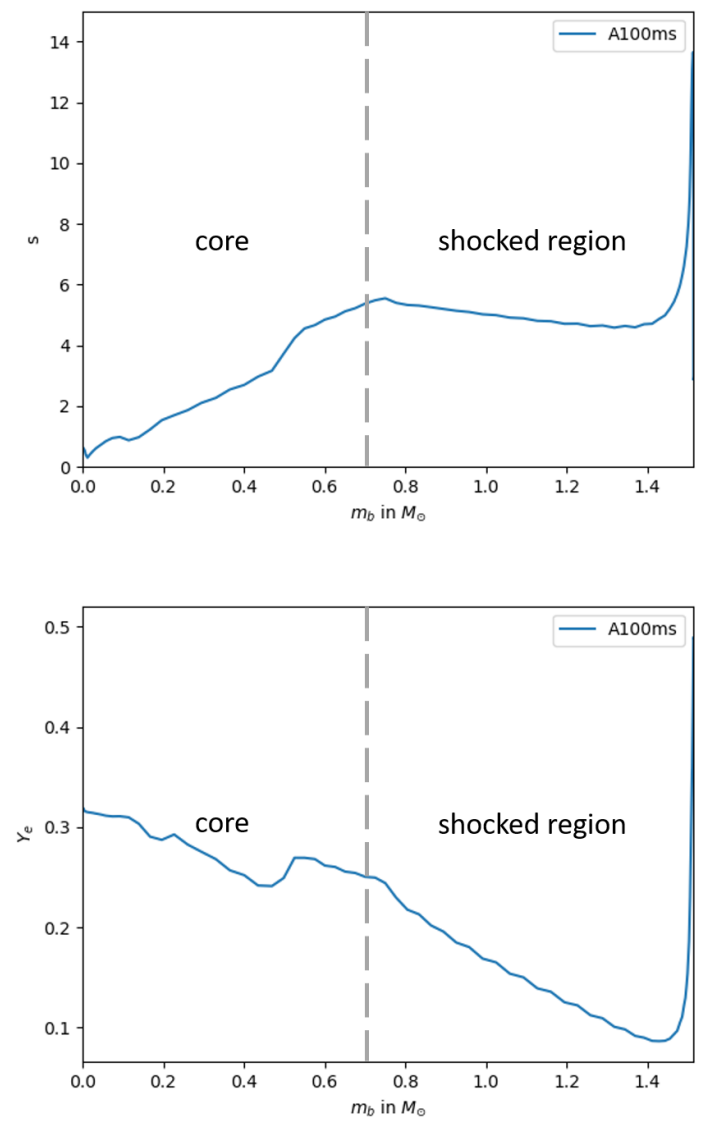

Figure 2. Entropy per baryon $s$ (top) and electron fraction $Y_{e}$ (bottom) as a function of the enclosed baryon mass $m_{b}$ for the configuration of profile A100ms from Fig. 1. The dashed line separates the inner core of the proto-neutron star from the shocked region.

where Eq. (6) corresponds to the projection along the $r$-axis of the conservation of the stress-energy tensor describing hydrostatic equilibrium. The final ingredient for the system constituted by Eqs. (4)-(6) to be closed is an EoS, that is a relation of the form $P=P(\mathcal{E})$ (equivalent to $P\left(n_{b}\right)$ introduced above). Since in a PNS thermal effects are not negligible and weak (beta-) equilibrium is not reached, a generic EoS for our case depends additionally, as stated in the Introduction, on the temperature $T$ and the composition of matter summarized by the electron fraction $Y_{e}$ (Oertel et al. 2017). Note that in presence of muons, the EoS would additionally depend on a muon fraction. Due to their higher masses, they contribute, however, only marginally to the EoS and we will neglect them here. A generic EoS then takes the form $P=P\left(\mathcal{E}, T, Y_{e}\right)$.

Consequently, in order to get an effective relation of the form $P=P(\mathcal{E})$ and integrate the TOV system to derive the PNS structure, it is necessary to assume some profiles $T(r)$ and $Y_{e}(r)$ or any equivalent ones inside the star. These profiles can be obtained from CCSN simulations and present a high variability, depending on the kind of progenitor that is considered for the supernova and on the time considered af- ter the formation of the PNS. Some examples are plotted in Fig. 1: temperature and electron fraction radial profiles coming from various CCSN simulations published in the literature are shown in order to give an idea of the existing uncertainties. To be more precise, the blue dashed-dotted curve corresponds to the profile at $0 \mathrm{~s}$ in Fig. 12 of Pons et al. (1999), the orange dashed curve to the model s 40 of Liebendörfer et al. (2004) and Fischer et al. (2009) at $400 \mathrm{~ms}$ after the bounce, the green dashed curves to profiles obtained using the CoCoNuT code (Dimmelmeier et al. 2005; Pascal et al. 2020) for a s15-type progenitor at $100 \mathrm{~ms}, 200 \mathrm{~ms}$ and $300 \mathrm{~ms}$ after the bounce, the magenta dotted curves to the profile for the model 1 su40 of Peres et al. (2013) and the red dashed curve to the model $\Lambda$-u 40 of Peres et al. (2013), both just before the collapse of the star into a black hole.

Since such profiles can a priori not be directly measured in a supernova event, their variability should be taken into account when making estimates about the structure of PNS. In other words, it is necessary, for any given EoS, to test many realistic profiles for $T$ and $Y_{e}$ in order to say something about the range of possible PNS structures that could arise for this EoS. In order to avoid performing a large number of computationally expensive CCSN simulations, we will use $p a-$ rameterized initial profiles at the bounce to investigate PNS structure.

These profiles are then evolved using a quasi-static evolution code (Pascal et al. (2021)): between two timesteps the electron fraction $Y_{e}$ and the entropy per baryon $s$ are evolved using equations (7)-(8) (see e.g. Pons et al. (1999) and Roberts \& Reddy (2017))

$$
\begin{aligned}
\frac{\mathrm{D} Y_{e}}{\mathrm{D} t} & =\frac{\mathrm{e}^{\Phi} S_{n}}{n_{b}} \\
\frac{\mathrm{D} s}{\mathrm{D} t} & =\frac{\mathrm{e}^{2 \Phi} S_{e}-\mathrm{e}^{\Phi} \mu_{e} S_{n}}{n_{b} T}
\end{aligned}
$$

where $\mu_{e}$ represents the electron chemical potential. Eq. (7) stems from lepton number conservation and Eq. (8) from energy conservation. For the latter, general thermodynamic relations have been used to express energy conservation in terms of entropy per baryon. The two source terms $S_{n}$ and $S_{e}$ are respectively the source of electrons and the source of energy issued from neutrino-matter interactions with a 3 -flavor neutrino transport. They are given by

$\begin{aligned} S_{n} & =-\frac{1}{c}\left(\Gamma_{\nu_{e}}-\Gamma_{\bar{\nu}_{e}}\right) \\ S_{e} & =-\frac{1}{c}\left(Q_{\nu_{e}}+Q_{\bar{\nu}_{e}}+4 Q_{\nu_{x}}\right)\end{aligned}$

with $\Gamma_{\nu_{e}}-\Gamma_{\bar{\nu}_{e}}$ the net electron neutrino creation rate which, by lepton number conservation, is related to the net change in electron number and $Q_{\nu}$ the energy loss rate induced by neutrino species $\nu$. Those rates are computed using a "Fast Multi-group Transport" (FMT) scheme (Müller \& Janka 2015). This scheme is based on a stationary approximation of neutrino transport and uses a two-ray approximation in areas with a high optical depth and a two-moment closure in areas with a low optical depth. Considered reaction rates are the same as in the original paper Müller \& Janka (2015).

For technical convenience, we choose here to parameterize the initial profiles that enter the PNS evolution code as the entropy per baryon as a function of the enclosed baryon mass $s\left(m_{b}\right)$ and the electron fraction $Y_{e}\left(m_{b}\right)$. In spherical 
symmetry, it is equivalent to use the radial coordinate $r$ or the enclosed baryon mass,

$$
m_{b}=4 \pi \int_{0}^{r} e^{\lambda} n_{b} m_{n} r^{2} \mathrm{~d} r .
$$

The latter presents the advantage of being a Lagrangian coordinate. Fig. 2 represents typical $s\left(m_{b}\right)$ and $Y_{e}\left(m_{b}\right)$ profiles obtained from CCSN simulations. Similar profiles can be found, e.g. in Fig. 1 of Roberts (2012). As can be seen, these profiles have the following characteristics: $s\left(m_{b}\right)$ first increases in the inner core of the star, then slightly decreases in a region corresponding to the shocked material and increases again very fast towards the surface. Here, the "inner core" should not be confused with the NS inner core, but be understood as the inner regions of the progenitor star. At bounce the shock has formed at the border of the inner core. Note that a more massive inner core (a wider region of increasing entropy per baryon in the $s\left(m_{b}\right)$ profile) implies that the kinetic energy of the shock is higher, such that it goes through more matter before exhaustion, which will result in a larger amount of shocked material (a wider region of decreasing entropy per baryon in the $s\left(m_{b}\right)$ profile). This means that the relative mass contained in both regions should be almost independent of the initial conditions (Janka 2012), as in the profile of Fig. 2. Second, $Y_{e}\left(m_{b}\right)$ is nearly constant $\sim 0.2-0.3$ in the inner core, then decreases more sharply in the shocked region and goes up again near the surface. We find that the following parameterizations of $s\left(m_{b}\right)$ and $Y_{e}\left(m_{b}\right)$ reproduce these main features.

$s\left(m_{b}\right)$ is modeled by a plateau followed by a Gaussian and a power-law divergence near the surface, with five parameters in total:

$$
\begin{aligned}
s\left(m_{b}\right)= & s_{c} \times\left[1+\exp \left(\frac{2 s_{\max }}{s_{c} \sigma_{s}}\left(m_{b}-m_{s}+2 \sigma_{s} / 3\right)\right)\right]^{-1} \\
& +s_{\max } \exp \left(-\frac{\left(m_{b}-m_{s}\right)^{2}}{2\left(\sigma_{s} / 2\right)^{2}}\right) \\
& +\frac{s_{\max }\left(M_{b}-m_{b}\right)^{-0.3}}{1+\exp \left(-\frac{2}{\sigma_{s}}\left(m_{b}-\left(m_{s}+2 \sigma_{s} / 3\right)\right)\right)}
\end{aligned}
$$

where $s_{c}$ is the central entropy per baryon, $s_{\max }$ the maximal entropy par baryon between inner core and shocked material, $\sigma_{s}$ the width of the Gaussian, $m_{s}$ the mass at which $s_{\max }$ is reached and $M_{b}$ the total baryon mass of the star. According to the remarks above, $m_{s}$ and $M_{b}-m_{s}$ should be of the same order for consistency with respect to the shock physics.

$Y_{e}\left(m_{b}\right)$ is modeled by a plateau followed by an inverse asymmetric Gaussian, with four parameters in total:

$$
\begin{aligned}
Y_{e}\left(m_{b}\right)= & Y_{0} \\
+ & \frac{Y_{c}-Y_{0}}{1+\exp \left(\frac{5}{2 \sigma_{Y}} \frac{Y_{\min }-Y_{0}}{Y_{c}-Y_{0}}\left(m_{b}-M_{b}+\frac{2 \sigma_{Y}}{3}\right)\right)} \\
+ & \left(Y_{\min }-Y_{0}\right) \exp \left(-\frac{\left(m_{b}-M_{b}\right)^{2}}{2\left(\sigma_{Y} / 2\right)^{2}}\right) \\
& \times \frac{1}{2}\left(1+\operatorname{erf}\left(-15 \frac{m_{b}-M_{b}}{\sigma_{Y} / \sqrt{2}}\right)\right)
\end{aligned}
$$

where "erf" refers to the error function and $Y_{c}$ is the central electron fraction, $Y_{0}=0.5, Y_{\min }$ the minimal $Y_{e}$ and $\sigma_{Y}$ the width of the Gaussian. Since the region where a depletion in $Y_{e}$ is observed, here described by the Gaussian, corresponds $[\mathrm{h}]$
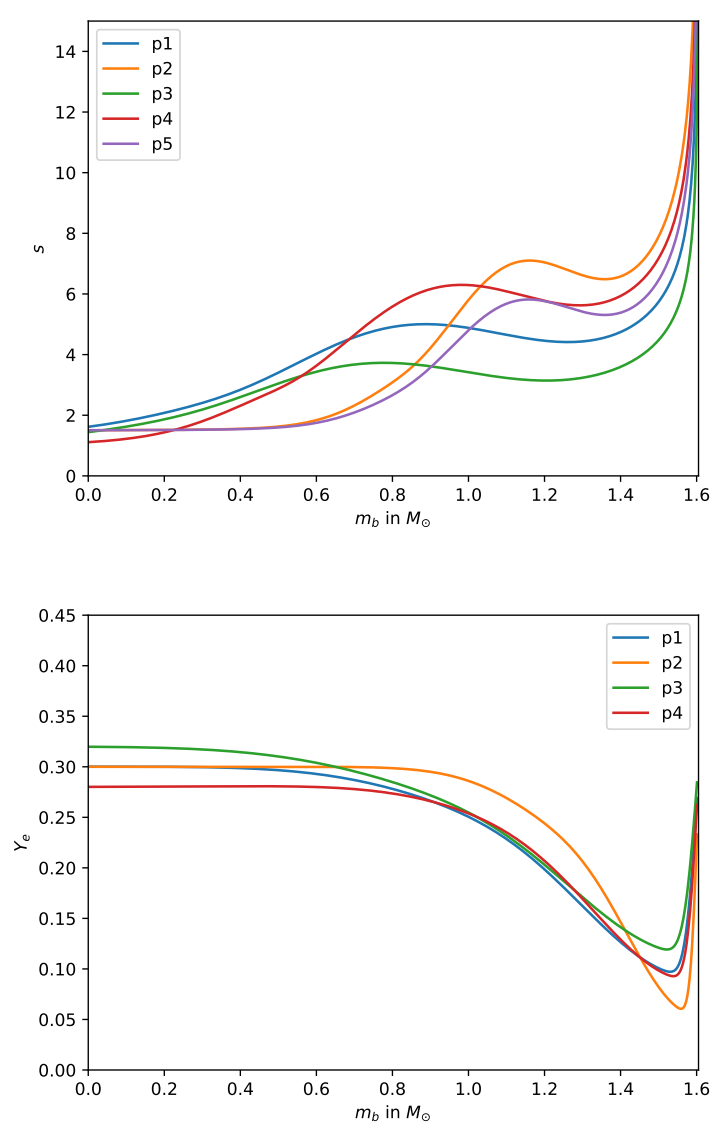

Figure 3. Entropy per baryon $s$ (top) and electron fraction $Y_{e}$ (bottom) as a function of the enclosed baryon mass $m_{b}$, for our chosen sets of parameterized profiles (12)-(13) defined at bounce, for a total baryon mass $M_{b}=1.6 M_{\odot} . Y_{e}$ profiles for the $\mathrm{p} 2$ and p5 parameterizations are identical.

to the shocked region (see Fig. 2), $\sigma_{Y}$ should be of the same order as $M_{b}-m_{s}$.

\section{NUMERICAL MODEL PARAMETERS AND RESULTS}

We present in this section the results of a comparison between several simulations of PNS structure during the first second of their post-bounce evolution varying the initial entropy and electron fraction profiles as well as the employed EoS model. Let us start with detailing the procedure applied for that comparison.

The initial profiles for $s\left(m_{b}\right)$ and $Y_{e}\left(m_{b}\right)$ at bounce are built upon the parameterizations discussed in the previous section, see Eqs. (12)-(13). The parameters are chosen such that the resulting profiles remain in reasonable agreement with results from CCSN. In particular, in order to respect the core-shock picture discussed above and illustrated in Fig. 2, $m_{s}$ and $\sigma_{Y}$ should be of the same order as $M_{b}-m_{s}$. For simplicity, we choose $\sigma_{Y}=M_{b}-m_{s}$. Note that $M_{b}$ is a parameter of both profiles, $s\left(m_{b}\right)$ and $Y_{e}\left(m_{b}\right)$, so that different 
$[\mathrm{h}]$
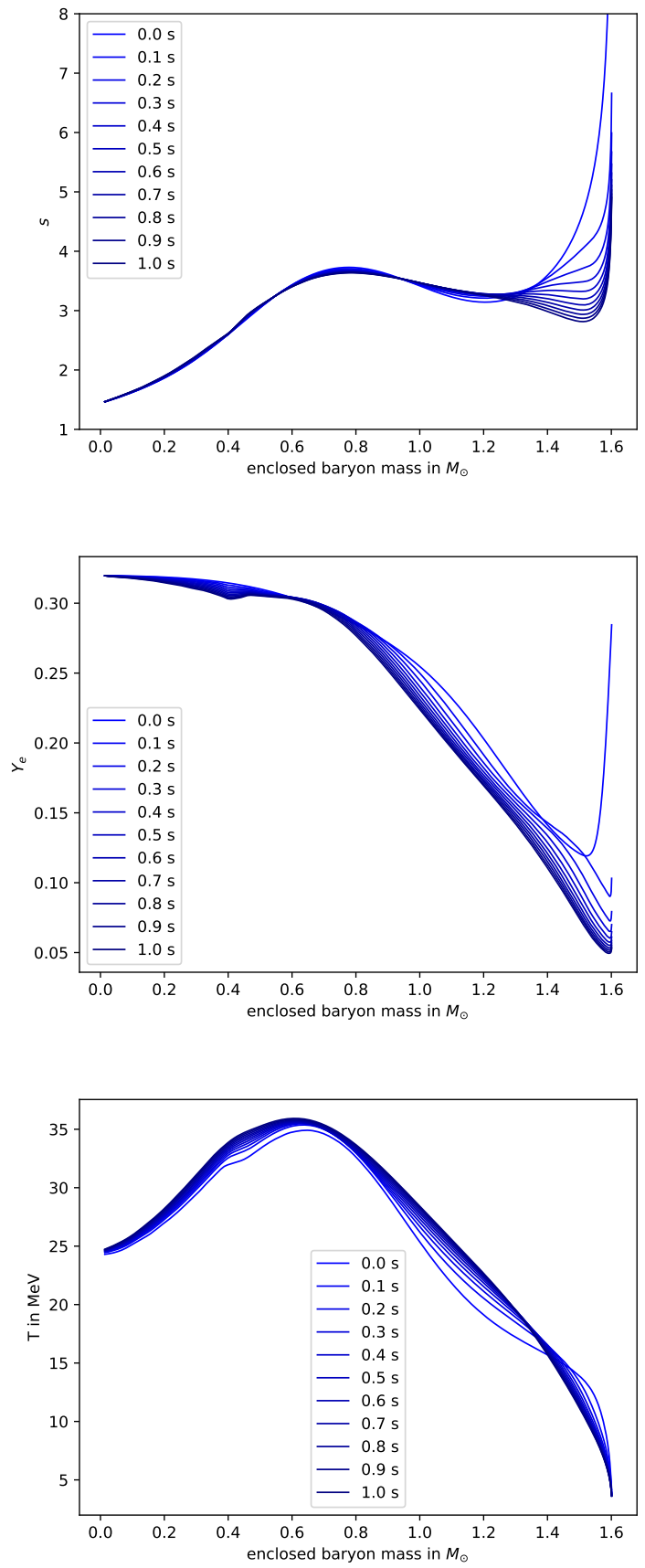

Figure 4. Entropy per baryon $s\left(m_{b}\right)$ (top), electron fraction $Y_{e}\left(m_{b}\right)$ (middle) and temperature $T\left(m_{b}\right)$ (bottom) profiles as a function of enclosed baryon mass $m_{b}$, for several post-bounce times (darker blue corresponds to later times) employing the initial profile p3 of Tab. 1, total baryon mass $M_{b}=1.6 M_{\odot}$ and the HS(DD2) EoS (Hempel \& Schaffner-Bielich 2010). The time evolution is computed within a quasi-static approach, see Sec. 2.

\begin{tabular}{lcccccc}
\hline Label & $s_{c}$ & $s_{\max }$ & $\sigma_{s}$ & $m_{s} / M_{b}$ & $Y_{c}$ & $Y_{\min }$ \\
\hline $\mathrm{p} 1$ & 1.3 & 4 & 0.7 & 0.5 & 0.26 & 0.1 \\
\hline $\mathrm{p} 2$ & 1.5 & 5.5 & 0.4 & 0.6875 & 0.3 & 0.06 \\
\hline $\mathrm{p} 3$ & 1.1 & 3 & 0.7 & 0.4375 & 0.32 & 0.12 \\
\hline $\mathrm{p} 4$ & 1 & 5 & 0.6 & 0.5625 & 0.28 & 0.1 \\
\hline $\mathrm{p} 5$ & 1.5 & 4.5 & 0.4 & 0.6875 & 0.3 & 0.06 \\
\hline
\end{tabular}

Table 1. Parameters defining for a given $M_{b}$ the five parameterizations labeled $\mathrm{p} 1$ to $\mathrm{p} 5$ of the initial $s\left(m_{b}\right)$ and $Y_{e}\left(m_{b}\right)$ profiles.

baryon masses correspond to different profiles. We say that two profiles obey the same parameterization if they have the same parameters, except for $M_{b}$ which can be chosen freely. $m_{s}$ is then given by the ratio $m_{s} / M_{b} \approx 1 / 2$. The values of the remaining free parameters for the five parameterizations considered here are detailed in Tab. 1. For each parameterization, three different total baryon masses $M_{b}$ will be considered: $1.6 M_{\odot}, 1.8 M_{\odot}$ and $2.0 M_{\odot}$. For illustration, the parameterized profiles are plotted in Fig. 3 for a total baryon mass $M_{b}=1.6 M_{\odot}$, the upper panel showing $s\left(m_{b}\right)$ and the lower one $Y_{e}\left(m_{b}\right)$. Profile $\mathrm{p} 1$ is thereby chosen to resemble the profiles of Fig. 2 and p2 those of Fig. 1 in Roberts (2012).

Then, with five parameterizations and three baryon masses, we end up with a total of 15 different initial profiles. These profiles are evolved during the first second postbounce for a given EoS using the quasi-static PNS evolution code (Pascal et al. 2021), see Section 2. Fig. 4 shows as an example the $s\left(m_{b}\right), Y_{e}\left(m_{b}\right)$ and $T\left(m_{b}\right)$ profiles in the PNS every $0.1 \mathrm{~s}$ post-bounce, for the parameterization $\mathrm{p} 3$ of Tab. 1, a total baryon mass $M_{b}=1.6 M_{\odot}$ and the HS(DD2) EoS (Hempel \& Schaffner-Bielich 2010).

Finally, with evolved profiles taken every 0.1 s post-bounce, we end up with 150 PNS profiles for each EoS. These profiles are used to solve the TOV system (4)-(6) and to derive the PNS structure at fixed baryon mass at each time. The surface of the PNS is thereby defined as in Torres-Forné et al. (2019a) at a fixed baryon density of $n_{b}=5.970 \times 10^{-5} \mathrm{fm}^{-3}$. We obtain in the end one point in the $\left(R_{\mathrm{PNS}}, M_{\mathrm{PNS}}\right)$ plane for each of the 150 PNS profiles.

Based on the results of the procedure described above, we first address the problem (P1), concerning the possibility, from the observation of mass and radius of a hot PNS in the first second post-bounce, to constrain the corresponding quantities of a cold neutron star. To take into account the uncertainty on the entropy and electron fraction in the PNS, the PNS structure is computed for the five parameterizations of initial profiles given in Tab. 1. In Fig. 5 are displayed the time evolution of 60 different PNS: our 15 different initial profiles with four different choices 
$[\mathrm{h}]$
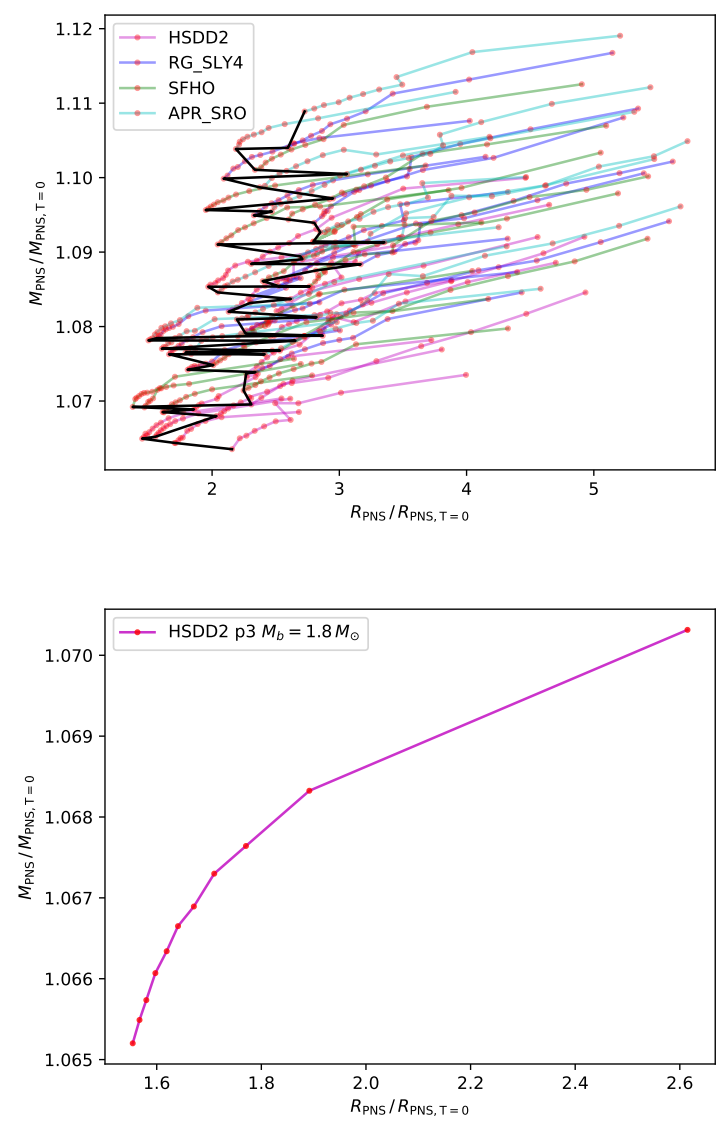

Figure 5. Top : PNS evolutions in the gravitational mass $\left(M_{\mathrm{PNS}}\right)$ radius $\left(R_{\mathrm{PNS}}\right)$ plane, normalized to the zero temperature values (subscript $T=0$ ), for the parameter sets $\mathrm{p} 1$ - p5 of Table 1. Different colors correspond to different EoS models: HS(DD2) (Hempel \& Schaffner-Bielich 2010, magenta), RG(SLy4) (Raduta \& Gulminelli 2019, blue), SFHo (Steiner et al. 2013b, green) and SRO(APR) (Schneider et al. 2019, cyan). Time runs from $0 \mathrm{~s}$ to $1 \mathrm{~s}$ post-bounce from right to left. The red dots indicate the points computed every $0.1 \mathrm{~s}$ and the black line the points for the PNS $1 \mathrm{~s}$ after the bounce. Bottom : same curve in the particular case of the HS(DD2) EoS, parameterization p3 of Tab. 1 and a total baryon $\operatorname{mass} M_{b}=1.8 M_{\odot}$.

of EoS models. These are HS(DD2) (Hempel \& SchaffnerBielich 2010, magenta), RG(SLy4) (Raduta \& Gulminelli 2019, blue), HS(SFHo) (Steiner et al. 2013b, green) and SRO(APR) (Schneider et al. 2019, cyan). The evolution is described in terms of the radius $R_{\mathrm{PNS}}$ and the mass $M_{\mathrm{PNS}}$, each one divided by the corresponding value for the cold configuration (label $T=0$ ), corresponding to a fully-evolved (cold, beta-equilibrated) NS.

It is apparent that for all parameterizations and EoSs, the PNS structure is still quite far from the cold configuration after $1 \mathrm{~s}$ of evolution. Quantitatively, we find that, after $1 \mathrm{~s}$, the mass is larger by about $6-10 \%$ and the radius by about $40-200 \%$ compared with the cold configuration. Note that, as far as the practical observation is concerned, because the $[\mathrm{h}]$

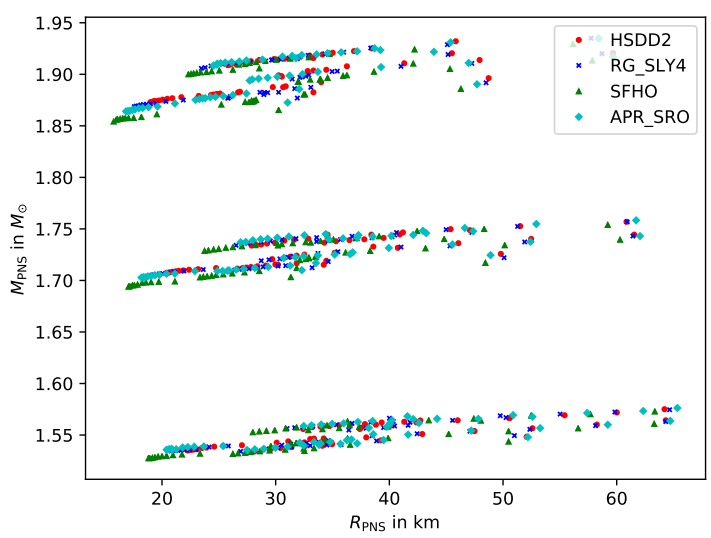

Figure 6. Points in the $\left(R_{\mathrm{PNS}}, M_{\mathrm{PNS}}\right)$-plane obtained from the evolution of the five initial parameterizations of Tab. (1), for three different total baryon masses, $M_{b}=1.6 M_{\odot}$ (bottom cloud), $M_{b}=1.8 M_{\odot}$ (middle cloud) and $M_{b}=2 M_{\odot}$ (top cloud). 4 different EoSs are used: HS(DD2) (Hempel \& SchaffnerBielich 2010, red circles), RG(SLy4) (Raduta \& Gulminelli 2019, blue crosses), SFHo (Steiner et al. 2013b, green triangles) and SRO(APR) (Schneider et al. 2019, cyan diamonds).

mass is almost constant ${ }^{1}$ during the first second of the PNS' life, its value is very little influenced by the time over which the observation is integrated, so that a good signal to noise ratio (SNR) is reachable in principle. On the contrary, integrating over a few tenths of a second would induce a large error on the radius measurement. All in all, our results imply that whereas measuring the mass of a PNS during the first second post-bounce would give an upper bound quite close to the actual value for the cold star, the obtained value of the radius is much less reliable. Indeed during the first few seconds of evolution of the PNS the shocked mantle cools down and shrinks very fast Prakash et al. (1997); Pons et al. (1999), such that the radius is evolving on a short timescale. As a consequence, the determination of $M_{\mathrm{PNS}} / R_{\mathrm{PNS}}^{2}$ proposed by Torres-Forné et al. (2019a) is not expected to give a reliable estimate of the same quantity in the cold configuration.

We now turn to the problem (P2), regarding the possibility, from the knowledge of $R_{\mathrm{PNS}}$ and $M_{\mathrm{PNS}}$, to constrain its EoS, which is fully dependent on three parameters. Although the answer would be yes if we were able to know, for each measured point at a given post bounce time, both $s$ and $Y_{e}$ profiles inside the PNS, the fact that they are not accessible implies that the actual answer depends on the influence of our limited knowledge of these profiles on $M_{\mathrm{PNS}}$ and $R_{\mathrm{PNS}}$. If the dispersion in $\left(R_{\mathrm{PNS}}, M_{\mathrm{PNS}}\right)$ due to the uncertainty in the profiles is smaller than the variation due to the difference induced by employing different EoS, then this measurement can in principle (provided a sufficient precision) constrain the EoS of the PNS. Fig. 6 shows the points obtained from the PNS evolution procedure for the four different EoS models considered before. In this figure, the condition for the

1 Except in the first tenth of a second, when the mass should increase if accretion is properly taken into account 
$[\mathrm{h}]$
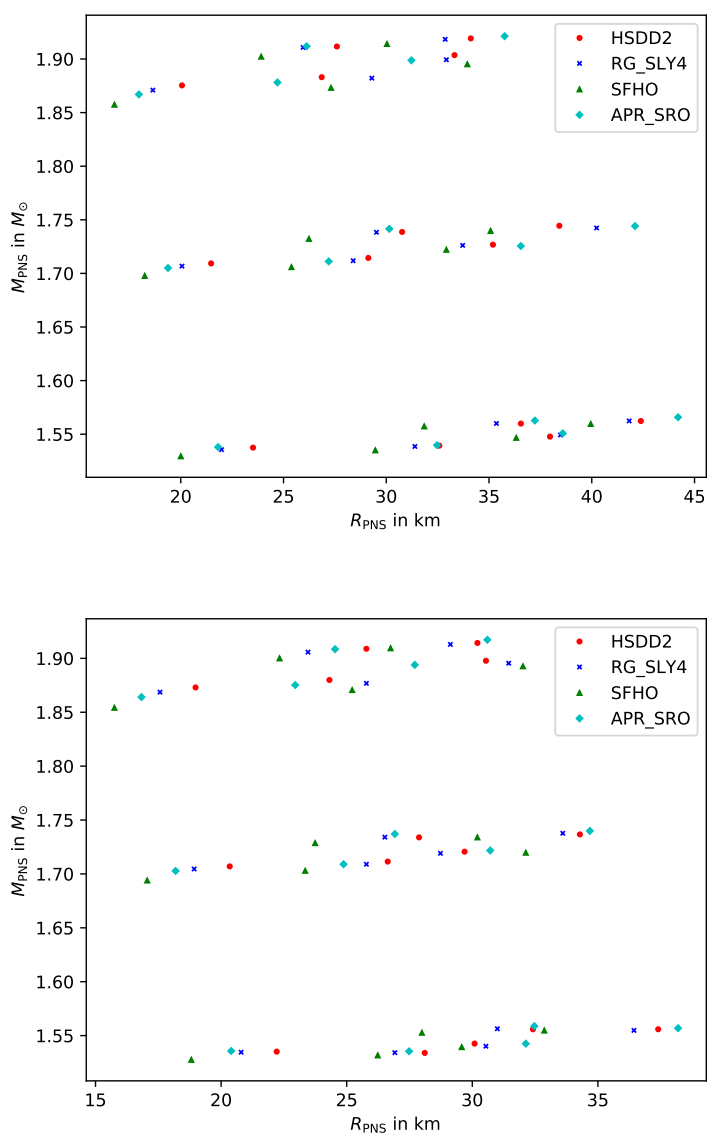

Figure 7. Same as Fig. 6, but only snapshots at $t=0.5 \mathrm{~s}$ (top) and $t=1 \mathrm{~s}$ (bottom) are taken from the time evolution.

$\left(R_{\mathrm{PNS}}, M_{\mathrm{PNS}}\right)$ measurement being able to discriminate between different EoS is that the clouds of points (corresponding to the various parameterizations for the initial $s$ and $Y_{e}$ profiles) corresponding to the different EoS models do not intersect. Actually, employing only four different EoS models, a positive answer to problem (P2) would actually be difficult to justify in this way. However, the clouds are largely mixed already for a small number of different EoS. We conclude that, measuring $R_{\mathrm{PNS}}$ and $M_{\mathrm{PNS}}$ alone can hardly constrain the EoS of the PNS. It is a priori possible to add some timing information to this picture, with the measure of the mass and radius at some given post-bounce times. To illustrate this purpose, Figs. 7 show snapshots of Fig. 6 at $0.5 \mathrm{~s}$ and $1 \mathrm{~s}$ after bounce. Here again, it is clear that the spreading of points (mainly along the $R_{\mathrm{PNS}}$ direction) due to the use of different entropy or electron fraction profiles is much larger than that coming from the various EoS models. This means in particular that, even if we can measure mass and radius of the PNS with additional timing information, little could be deduced about the underlying EoS.

In this context, it it is interesting to ask which of the uncertainty on entropy or on electron fraction has the largest influence on the large spreading in the $\left(M_{\mathrm{PNS}}, R_{\mathrm{PNS}}\right)$-plane responsible for the negative answer to (P1) and (P2), or whether they both contribute equally. To answer that question, we have introduced four additional parameterizations based on p1: two with a modified entropy keeping the electron fraction profile the same, and two with modified electron fraction and the same entropy profile. For the former, we vary $s_{c}$ and $s_{\max }$ by $\pm_{20}^{50} \%$ and $\pm 50 \%$, respectively, compared with the $\mathrm{p} 1$ entropy profile. These variations remain still in reasonable agreement with results from simulations. For the latter, we use two rather extreme profiles, one with a $Y_{c}$ and a $Y_{\min }$ increased by roughly $15 \%$ and $50 \%$ respectively and another one reducing $Y_{c}$ and $Y_{\min }$ by $30 \%$ and $70 \%$, respectively. Repeating the same exercise as before, i.e. following the evolution of the PNS over $1 \mathrm{~s}$ with these additional initial profiles and the same four different EoS models and analysing the obtained distribution in $M_{\mathrm{PNS}}$ and $R_{\mathrm{PNS}}$, the conclusion is rather obvious. Varying the $Y_{e}$-profile, even in an extreme way, we observe only a limited dispersion of points. As far as $M_{\mathrm{PNS}}$ is concerned, it is less than a few percent and for $R_{\text {PNS }}$, of the order of $10 \%$. On the contrary, the different entropy profiles induce a large uncertainty, in particular for the radii which spread between roughly 15 and $60 \mathrm{~km}$. Taking snapshots at given post-bounce times does not alter the conclusion that mainly the uncertainty in the entropy profile of the PNS prevents us from obtaining information about the EoS and cold neutron star properties from the observation of the PNS' mass and radius. This finding can be understood from the fact that small variations in entropy per baryon induce large variations in pressure and energy density of matter in particular at lower densities, i.e. in the outer regions of the star, and that radii are very sensitive to these variations. That means, if we want to improve the situation and be able to extract information from the measurement of PNS mass and radii, we should better constrain the entropy profiles in the PNS.

It should be mentioned here, too, that the PNS is very likely unstable to convection, flattening the entropy per baryon profile in the shocked region (see e.g. the discussion in Roberts \& Reddy 2017). This flattening is not reproduced by our profiles and the simulated PNS evolution does not treat convection. Including the latter would certainly change our results quantitatively, but the main result of our study remains true: without considerably reducing the uncertainty in entropy profiles, no useful information on EoS and cold NS mass and radius can be obtained from the corresponding quantities of a PNS.

\section{SUMMARY AND DISCUSSION}

As it seems to be possible to infer from future GW observations the mass and the radius of a newly born PNS, we have investigated here what information these data could bring about the nuclear EoS for NSs (one-parameter EoS) or for PNSs (3-parameter EoS). Thermal and composition effects cannot be neglected within a PNS. In addition, temperature and electron fraction in a PNS evolve with time and its mass and radius accordingly. We have thus considered neutrino cooling of the PNS using a quasi-static approach (Pascal et al. 2021) and determined entropy and electron fraction profiles in the PNS at several instants during the first second of postbounce evolution. Based on these profiles, we have built hydrostatic PNS models at each step giving mass and radius. 
From CCSN simulations it appears that large uncertainties exist as far as entropy and electron fraction profiles at early post-bounce are concerned. These uncertainties result, among others, from different progenitor structure, uncertainties on weak reactions during infall, the employed EoS and convection in the newborn PNS. In order to take these uncertainties into account, we have set up analytic parameterized profiles for both quantities ( $s$ ans $Y_{e}$ ), that can mimic most of the simulated profiles from the literature, enabling us to generate a large set of physically relevant evolutionary models of PNSs, in the first second after the bounce.

The study of this set of models showed that, after $1 \mathrm{~s}$ of evolution, the value of the radius is still far from the corresponding value of a cold NS. This indicates that the knowledge of PNS properties soon after the bounce cannot give much information on the properties of the resulting cold NS (question (P1)). Additionally, a large dispersion of evolutionary curves of PNS in the $M_{\mathrm{PNS}}, R_{\mathrm{PNS}}$ plane and, above all, a mixing of points coming from various EoSs is observed. Conversely, this means that, even with some timing information (as illustrated by Figs. 6 and 7), it is difficult to disentangle evolutionary tracks of models with different EoSs. This also leads to a negative answer to the question (P2), about the possibility of inferring the EoS of the PNS from its masses and radii.

Of course, there are several limitations to this study. First, the quasi-static approach may not be the most accurate, but we believe that, as discussed in previous works Pons et al. (1999); Villain et al. (2004), such an approximation is under control and is not the main source of uncertainties in our results. Another point which merits discussion is the choice of parameterized profiles. The choices done here, with formulas in Eqs. (12)-(13), may be regarded as too broad, giving too much freedom in the dispersion of points in Figs. 5 and 6. However, our choices of parameters listed in Tab. 1 are such that they should reproduce previous results from CCSN simulations. As these simulations include not fully controlled ingredients, in particular initial data for the progenitor stars, it is important to consider a general set of profiles, unless future studies can bring techniques to determine temperature and electron fraction profiles in CCSN from astrophysical observable features. Last but not least, a limitation of our study is the fact that we have considered the evolution of the PNS only during the first second after its birth. In particular concerning the problem (P1) about information of cold NS, the situation would of course be quite different if longer evolution times were considered. The particular value of $1 \mathrm{~s}$ postbounce is justified by CCSN simulations showing that during this period, a quasi-stationary situation is achieved for the stalled accretion shock and instability-induced perturbations of the PNS. It is not clear to what extent strong-enough GW can be emitted later, and therefore whether it is still possible to determine $M_{\mathrm{PNS}}$ and $R_{\mathrm{PNS}}$ at later stages. This point would certainly be worth exploring more in details, as later information would certainly be more decisive in order to get insights on nuclear matter properties. A possibility in this direction is evoked in Gallo Rosso et al. (2018), estimating the PNS radius at about 6 -10s after bounce from the neutrino signal. However, for the moment the uncertainties entering the neutrino signal, in particular those related to neutrino transport in the newborn hot PNS and to neutrino-matter interactions, render a reliable extraction of the PNS radius prohibitive.

\section{ACKNOWLEDGEMENTS}

We warmly thank Pablo Cerdá-Durán and Alejandro TorresForné for enlightening discussions, and Francesca Gulminelli for interesting suggestions. The research leading to these results has received funding from the PICS07889; it was also partially supported by the Observatoire de Paris through the action fédératrice "PhyFog".

\section{REFERENCES}

Abbott B. P., et al. LIGO Scientific Collaboration Virgo Collaboration 2018, Phys. Rev. Lett., 121, 161101

Capano C. D., et al., 2020, Nature Astron., 4, 625

De S., Finstad D., Lattimer J. M., Brown D. A., Berger E., Biwer C. M., 2018, Phys. Rev. Lett., 121, 091102

Dexheimer V., de Oliveira Gomes R., Schramm S., Pais H., 2019, J. Phys. G, 46, 034002

Dimmelmeier H., Novak J., Font J. A., Ibáñez J. M., Müller E., 2005, Phys. Rev. D, 71, 1

Fischer T., Whitehouse S. C., Mezzacappa A., Thielemann F. K., Liebendörfer M., 2009, A\&A, 499, 1

Gallo Rosso A., Abbar S., Vissani F., Volpe M. C., 2018, JCAP, 12,006

Güven H., Bozkurt K., Khan E., Margueron J., 2020, Phys. Rev. C, 102, 015805

Haensel P., Potekhin A. Y., Yakovlev D. G., 2007, Neutron Stars 1 : Equation of State and Structure. ASSL Vol. 326, Springer

Hempel M., Schaffner-Bielich J., 2010, Nuclear Physics A, 837, 210

Hinderer T., Lackey B. D., Lang R. N., Read J. S., 2010, Phys. Rev. D, 81, 123016

Janka H.-T., 2012, Ann. Rev. Nucl. Part. Sci., 62, 407

Kramer M., et al., 2006, Science, 314, 97

Liebendörfer M., Messer O. E. B., Mezzacappa A., Bruenn S. W., Cardall C. Y., Thielemann F. K., 2004, The Astrophysical Journal Supplement Series, 150, 263

Malik T., Agrawal B., De J., Samaddar S., Providência C., Mondal C., Jha T., 2019, Phys. Rev. C, 99, 052801

Miller M. C., et al., 2019, Astrophys. J. Lett., 887, L24

Müller B., Janka H. T., 2015, MNRAS, 448, 2141

Oertel M., Hempel M., Klähn T., Typel S., 2017, Rev. Mod. Phys., 89, 015007

Ozel F., Baym G., Guver T., 2010, Phys. Rev. D, 82, 101301

Ozel F., Psaltis D., Guver T., Baym G., Heinke C., Guillot S., 2016, Astrophys. J., 820, 28

Pascal A., Giraud S., Fantina A. F., Gulminelli F., Novak J., Oertel M., Raduta A. R., 2020, Phys. Rev. C, 101, 015803

Pascal A., Oertel M., Novak J., Mancini M., 2021, Proto-neutron star cooling with improved neutrino-nucleon interactions, In preparation

Peres B., Oertel M., Novak J., 2013, Phys. Rev. D, 87, 043006

Pons J. A., Reddy S., Prakash M., Lattimer J. M., Miralles J. A., 1999, ApJ, 513, 780

Prakash M., Bombaci I., Prakash M., Ellis P. J., Lattimer J. M., Knorren R., 1997, Phys. Rept., 280, 1

Raduta A. R., Gulminelli F., 2019, Nucl. Phys., A983, 252

Riley T. E., et al., 2019, ApJ Lett., 887, L21

Roberts L. F., 2012, ApJ, 755, 126

Roberts L. F., Reddy S., 2017, in Alsabti A. W., Murdin P., eds, , Handbook of Supernovae. Springer International Publishing AG, p. 1605, doi:10.1007/978-3-319-21846-5 5 
Schneider A. S., Constantinou C., Muccioli B., Prakash M., 2019, Phys. Rev. C, 100, 025803

Steiner A. W., Lattimer J. M., Brown E. F., 2010, Astrophys. J., 722,33

Steiner A. W., Lattimer J. M., Brown E. F., 2013a, Astrophys. J. Lett., 765, L5

Steiner A. W., Hempel M., Fischer T., 2013b, The Astrophysical Journal, 774, 17

Torres-Forné A., Cerdá-Durán P., Passamonti A., Font J. A., 2018, MNRAS, 474, 5272

Torres-Forné A., Cerdá-Durán P., Obergaulinger M., Müller B., Font J. A., 2019a, Phys. Rev. Lett., 123, 051102

Torres-Forné A., Cerdá-Durán P., Passamonti A., Obergaulinger M., Font J. A., 2019b, MNRAS, 482, 3967

Villain L., Pons J. A., Cerdá-Durán P., Gourgoulhon E., 2004, Astron. Astrophys., 418, 283

Wex N., Kramer M., 2020, Universe, 6, 156

This paper has been typeset from a $\mathrm{TEX}_{\mathrm{E}}$ /LATEX file prepared by the author. 